\title{
Hydroxyethyl starch for fluid resuscitation in critically ill patients
}

\author{
Sean M. Bagshaw, MD • Lakhmir S. Chawla, MD
}

Received: 11 November 2012/ Accepted: 10 April 2013/Published online: 20 April 2013

(c) Canadian Anesthesiologists' Society 2013

\section{Article appraised}

Myburgh JA, Finfer S, Bellomo R, et al. Hydroxyethyl starch or saline for fluid resuscitation in intensive care. N Engl J Med 2012: 367: 1901-11.

\section{Structured Abstract}

Background: Intravenous fluid therapy is one of the most frequent interventions provided to patients in the intensive care unit; however, the type of fluid (i.e., crystalloid or colloid) used for resuscitation remains controversial. The most common type of colloid administered to resuscitate critically ill patients is hydroxyethyl starch (HES); however, its safety and efficacy have not been rigorously evaluated in large pragmatic randomized trials, and emerging data have accumulated to question its potential for toxic adverse effects.

Objective: To evaluate the efficacy and safety of HES for fluid resuscitation in critically ill patients with a focus on survival and kidney function.

This article is accompanied by an editorial. Please see Can J Anesth 2013; 60: this issue.

\section{S. M. Bagshaw, MD ( $\square)$}

Division of Critical Care Medicine, Faculty of Medicine and Dentistry, University of Alberta, 3C1.12 Walter C. Mackenzie Centre, 8440-122 ST NW, Edmonton, AB T6G 2B7, Canada e-mail: bagshaw@ualberta.ca

L. S. Chawla, MD

Department of Anesthesiology and Critical Care Medicine, George Washington University Medical Center, Washington, DC, USA
Design: Multicentre (32 sites in Australia and New Zealand) blinded randomized controlled parallel-group trial.

Methods: Seven thousand eligible adult patients (age $\geq 18 \mathrm{yr}$ ) admitted to an intensive care unit and judged by their treating clinician to require fluid resuscitation were included in the study. Study treatment allocation used encrypted Web-based randomization stratified by site and an admission diagnosis of trauma.

Intervention: Randomized patients were assigned to receive either $6 \%$ HES with a molecular weight of $130 \mathrm{kD}$ and molar substitution ratio of $0.4\left(130 / 0.4\right.$; Voluven ${ }^{\circledR}$, Fresenius Kabi) in $0.9 \%$ sodium chloride or $0.9 \%$ sodium chloride (saline) in indistinguishable Freeflex $500 \mathrm{~mL}$ bags until intensive care unit (ICU) discharge, death, or 90 days after randomization. According to registration guidelines, the study fluid was administered to a maximum dose of $50 \mathrm{~mL} \cdot \mathrm{kg}^{-1}$ body weight per day and followed, if necessary, by open-label saline during the remaining 24 -hr period.

Measurements: The primary efficacy outcome was death within 90 days after randomization. The key secondary outcomes were incidence of acute kidney injury (AKI), defined by the RIFLE (Risk, Injury, Failure, Loss, Endstage) criteria; treatment with renal replacement therapy (RRT); development of new organ dysfunction, defined by the sequential organ failure assessment score; duration of mechanical ventilation; duration of RRT; cause-specific mortality; and adverse events. Tertiary outcomes were ICU and hospital lengths of stay and ICU and hospital mortality. The primary outcome was evaluated across six a priori defined subgroups: urine output criteria for AKI; presence of sepsis; presence of trauma, with or without traumatic brain injury; acute physiology and chronic health evaluation (APACHE) score $\geq 25$; and receipt of HES prior to randomization. 
Main results: The HES and saline groups had similar characteristics at baseline. The average age was $63 \mathrm{yr}$, $60.4 \%$ of patients were male, and $42.7 \%$ were admitted to the ICU after surgery (54.7\% after elective surgery). The median [interquartile range] APACHE II score was 17 [12.0-23.0] with a score $\geq 25$ in $18.2 \%$. Sepsis and trauma were primary diagnoses in $28.8 \%$ and $7.9 \%$ of patients, respectively. Mechanical ventilation was received by $64.5 \%$ of patients, vasopressor therapy by $45.8 \%$, and HES fluid prior to randomization by $15.1 \%$. Enrolment occurred approximately $11 \mathrm{hr}$ after ICU admission. During the first four days after randomization, the mean (standard deviation) study fluid received by the HES group was less when compared with the saline group [526 (425) $\mathrm{mL} \cdot$ day $^{-1} v s$ 616 (488) $\mathrm{mL} \cdot \mathrm{day}^{-1}$, respectively; $\left.P<0.001\right]$. Mortality at 90 days was $18.0 \%$ in patients receiving $\operatorname{HES}(597 / 3,315)$ and $17.0 \%$ in those receiving saline $(566 / 3,336)$ (relative risk [RR] for HES, 1.06; 95\% confidence interval (CI), 0.96 to $1.18 ; P=0.26$ ). There was no significant difference in 90-day mortality across the six a priori defined subgroups. Renal replacement therapy was received in $7.0 \%$ of patients in the HES group $(235 / 3,352)$ and $5.8 \%$ of patients in the saline group $(196 / 3,376)$ (RR for HES, 1.21; 95\% CI, 1.00 to $1.45 ; P=0.04)$. In the HES and saline groups, RIFLE - Injury occurred in $34.6 \%$ and $38.0 \%$ of patients, respectively $(P=0.005)$, and RIFLE - Failure occurred in $10.4 \%$ and $9.2 \%$ of patients, respectively $(P=0.12)$. There were no differences in mortality in ICU, in hospital, or at 28 days. Hydroxyethyl starch was associated with a decrease in new cardiovascular organ failure compared with saline (36.5\% vs $39.9 \%$, respectively; RR 0.91 ; $95 \%$ CI, 0.84 to $0.99 ; P=0.03$ ) and an increase in new hepatic organ failure compared with saline $(1.9 \%$ vs $1.2 \%$, respectively; RR 15.6 ; $95 \%$ CI, 1.03 to $2.36 ; P=0.03$ ). There were no differences between HES and saline for days in ICU or hospital or for duration of mechanical ventilation or RRT. Hydroxyethyl starch was associated with more adverse events compared with saline $(5.3 \% \mathrm{vs}$ $2.8 \%$, respectively; RR 1.86; 95\% CI, 1.46 to 2.38; $P<0.001)$. Adverse events were predominantly accounted for by pruritis and skin rash.

Conclusion: In critically ill patients receiving fluid resuscitation, there was no significant difference in 90-day mortality between $6 \%$ HES (130/0.4) or saline. Even so, more patients who received resuscitation with HES were treated with RRT and experienced adverse events.

Source of funding: The trial was funded by grants from the National Health and Medical Research Council of Australia and the New South Wales Ministry of Health. Fresenius Kabi supplied the study fluids. The funding agencies and industry had no involvement in the design, conduct, data collection, analyses, or manuscript preparation.

\section{Commentary}

Intravenous fluid therapy, aimed largely at restoring intravascular volume deficits, is arguably one of the most common interventions administered to critically ill patients. ${ }^{1}$ Nevertheless, there remains long-standing controversy about the ideal fluid type for use during resuscitation, i.e., crystalloids or colloids. ${ }^{2}$ Recent observational data imply that colloids, predominantly synthetic hydroxyethyl starches (HES), are the most common fluid type used for resuscitation in critically ill patients. ${ }^{1}$ In Canada, HES solutions are frequently administered for resuscitation in septic shock. ${ }^{3}$ In a recent multicentre study, colloid therapy, predominantly HES, was used in an estimated $40 \%$ of all fluid resuscitation episodes. ${ }^{1}$

\section{Current state of the literature and study relevance}

Numerous experimental studies have provided a physiological rationale for the preferential use of a colloid over crystalloid therapy, with an emphasis on HES for resuscitation in septic shock and in states of acute stress (i.e., major trauma, perioperative). Hydroxyethyl starch solutions have been shown to attenuate the acute inflammatory response, ${ }^{4-6}$ mitigate endothelial barrier dysfunction and vascular leak, ${ }^{6,7}$ and preserve intestinal barrier function. ${ }^{4}$ Small clinical trials have suggested HES solutions are also superior for resuscitation of the microcirculation in sepsis, ${ }^{8}$ contribute to more rapid hemodynamic stabilization and shock reversal, and require significantly less fluid to restore intravascular volume. ${ }^{9,10}$

Even so, the literature has been dominated largely by small lower-quality randomized trials that have precluded a clear appraisal of potential survival benefit and the risk of toxicity associated with the use of HES for resuscitation. ${ }^{11,12}$ Nonetheless, accrued data from these randomized trials have raised serious concern about the potential kidney-related toxic effects of HES leading to greater utilization of renal replacement therapy (RRT). ${ }^{9,13-15}$ There has been suggestion that HES solutions with a lower molecular weight and a lower degree of molar substitution have an improved safety profile with regard to bleeding complications and acute kidney injury (AKI); however, findings have been inconsistent. ${ }^{9,10,16-20}$ Experimental data have shown that these newer generation HES solutions can still accumulate in tissues within six hours of administration, including in the liver, kidney, lung, spleen, and lymph nodes. $^{21}$

Accordingly, it is particularly relevant that two large well-designed and conducted randomized controlled trials have recently been published evaluating the efficacy and safety of newer HES solutions (concentration 6\%; molecular weight $130 \mathrm{kD}$; molar substitution $0.40-0.42$ ) for fluid 
resuscitation in critically ill patients. ${ }^{22,23}$ These trials endeavour to answer the critical unresolved question: When these HES solutions are compared with crystalloid solutions, do they contribute to more rapid hemodynamic stabilization and shock reversal while requiring less fluid administration, and do these physiological benefits translate into improved patient outcomes?

The recently published Scandinavian Starch for Severe Sepsis/Septic Shock (6S) trial was a multicentre randomized controlled trial comparing 6\% HES (130/0.42) in Ringer's acetate with Ringer's acetate for fluid resuscitation (up to $33 \mathrm{~mL} \cdot \mathrm{kg}^{-1} \cdot \mathrm{day}^{-1}$ ) on the primary end point of 90-day all-cause mortality or dialysis dependence in 798 critically ill patients with septic shock. ${ }^{23}$ Participants were enrolled after their initial resuscitation, and the trial found no significant differences in the total volume of study fluid administered between groups. As such, this trial was not able to show greater efficacy in terms of faster hemodynamic stabilization with HES compared with Ringer's acetate. Nevertheless, in patients allocated to receive HES, the rate of death or RRT at 90-days was significantly higher compared with those receiving Ringer's acetate $(51 \% \mathrm{vs}$ $43 \%$ respectively; relative risk [RR] $1.17 ; 95 \%$ confidence interval (CI), 1.01 to $1.36 ; P=0.03$ ), and the utilization of RRT was also significantly higher $(22 \%$ vs $16 \%$, respectively; RR $1.35 ; 95 \% \mathrm{CI}, 1.01$ to $1.80 ; P=0.04)$. These findings appear to confirm the increased risk of toxicity evident in prior trials focused on critically ill patients with sepsis. ${ }^{13,15}$ Moreover, the observed hazard of death with HES was delayed (30-90 days), implying that prior trials of HES with short-term follow-up may not have been able to detect the risk.

Similar to the $6 \mathrm{~S}$ trial, the Crystalloid Versus Hydroxyethyl Starch Trials (CHEST) study enrolled patients after admission to ICU (median $\sim 11 \mathrm{hr}$ after admission). Contrary to $6 \mathrm{~S}$, though, the CHEST study showed that patients allocated to HES received significantly less study-related and non-study-related fluid compared with saline, contributing to a less positive fluid balance in the four days after randomization, perhaps implying greater efficacy for HES compared with saline. This interpretation is further strengthened by less new cardiovascular organ failure occurring in patients allocated to HES than in those allocated to saline.

Importantly, while the CHEST study found HES-treated patients had similar rates of death at 90 days compared with saline-treated patients, the range in estimated effect size favoured saline $(-4 \%$ to $18 \%)$. Coupled with the observed consistency across all six a priori subgroups evaluated, the study results would seem to reinforce a greater potential for downstream harm. Moreover, similar to the $6 \mathrm{~S}$ trial, the CHEST study found a greater utilization of RRT with HES when compared with saline. The paradoxical observation of a reduced incidence of AKI with HES compared with saline appears largely driven by differences in the creatinine and urine output components of the RIFLE (Risk, Injury, Failure, Loss, End-stage) classification for AKI. In a post hoc analysis, the CHEST study found a dose-response increase in risk of AKI with HES compared with saline when evaluating the RIFLE creatinine component; whereas, this was not evident for the RIFLE - urine output component. This may imply that HES better preserved urine output at the expense of declines in glomerular filtration when compared with saline.

\section{Analysis of methodology}

The CHEST study was designed as a large-scale pragmatic trial to evaluate the efficacy and safety of $6 \%$ HES in saline compared with saline for fluid resuscitation in a heterogeneous cohort of critically ill patients. The study was performed with a high degree of internal validity, including a priori publication of the trial protocol and a statistical analysis plan. ${ }^{24}$ Despite these virtues, the CHEST study has potential limitations worth discussion. First, while patients had to fulfill at least one of six pre-specific criteria (supplementary Table $\mathrm{S} 1 \mathrm{~A})^{24}$ as a requirement for fluid administration, the trial did not specify additional aspects of fluid management, such as the rate or total volume coupled with end points of resuscitation. Instead, these aspects were determined at the discretion of treating clinicians. Second, the protocol did not provide specific criteria for RRT initiation or the administration of blood products. As mentioned previously, the HES-treated patients had higher utilization of both RRT and blood products during the first four days after randomized compared with the saline-treated patients. Nevertheless, the potential influence of these limitations on the observed differences in effects are likely diminished in such a "realworld" pragmatic trial given that study allocation was concealed and the intervention was blinded. Third, prior data have suggested there is a dose-response increase in the risk of kidney toxicity associated with HES. ${ }^{15}$ In the CHEST study, patients were permitted to receive up to $50 \mathrm{~mL} \cdot \mathrm{kg}^{-1} \cdot \mathrm{day}^{-1}$ of study fluid, and then patients received open-label saline for the remainder of the $24 \mathrm{hr}$ period in accordance with the safety data filed for $6 \%$ HES with the Therapeutic Goods Administration of Australia and the New Zealand Medicines and Medical Devices Safety Authority. While data on the daily administered dose $\left(\mathrm{mL} \cdot \mathrm{kg}^{-1} \cdot \mathrm{day}^{-1}\right)$ are not presented, patients appeared to have received considerably less than this volume [mean (standard deviation) daily average of HES was 526 (425) $\mathrm{mL} \cdot \mathrm{day}^{-1}$ in the first four days]. Finally, while RRT was received by more HES-treated patients, the incidence of 
AKI, defined by RIFLE categories $\mathrm{R}$ and $\mathrm{I}$, were paradoxically more common among the saline-treated patients. It was only after a post hoc analysis of the creatinine and urine output components of the RIFLE criteria that there was evidence implying a greater risk of toxicity by increases in creatinine rather than urine output.

Relevance to clinical practice (external validity)

The CHEST study is likely to be widely generalizable to heterogeneous cohorts of critically ill patients. Even so, the CHEST study was characterized by a lower than expected rate of death, a relatively large subgroup of admissions after elective surgery $(\sim 23-24 \%)$, and an overall lower illness severity (APACHE II score $\sim 17$ ), where only approximately $65 \%$ of patients received mechanical ventilation and $45 \%$ received vasopressors. For context, in the 6S trial, $84 \%$ of patients had shock at baseline, only $10.3 \%$ were admitted after elective surgery, and observed mortality was approximately 2.5 times greater.

\section{Clinical perspective}

The CHEST study, coupled with the $6 \mathrm{~S}$ trial and other accumulated data, ${ }^{9,13,15}$ would present a reasonable argument to avoid the use of HES solutions for fluid resuscitation in critically ill patients. While there may be immediate physiological benefit for HES solutions over crystalloids, these are associated with delayed kidney toxicity and less favourable clinical outcomes. Whether some forms of HES are less nephrotoxic than others remains unclear, but the aggregate clinical trial data suggest a class effect of nephrotoxicity for HES. In addition, a unit of HES $(500 \mathrm{~mL})$ is approximately 45 times more expensive than a $1 \mathrm{~L}$ unit of crystalloid, signifying no costeffectiveness. Accordingly, the use of HES for fluid resuscitation in critically ill patients would not appear to be justified given evidence of no clear survival benefit, increased risk of harm, and excessive relative cost compared with crystalloid solutions. It is likely that these findings will have increasingly important implications for the use of HES for fluid resuscitation in perioperative medicine. $^{25}$

Acknowledgements S.M.B. is supported by a Canada Research Chair in Critical Care Nephrology and Clinical Investigator Award from Alberta Innovates - Health Solutions.

Financial disclosures The authors have no financial disclosures of interest to declare.

Conflicts of interest None declared.

\section{References}

1. Finfer $S$, Liu B, Taylor $C$, et al. Resuscitation fluid use in critically ill adults: an international cross-sectional study in 391 intensive care units. Crit Care 2010; 14: R185.

2. Perel P, Roberts I. Colloids versus crystalloids for fluid resuscitation in critically ill patients. Cochrane Database Syst Rev 2012; 6: CD000567.

3. McIntyre LA, Hebert PC, Fergusson D, Cook DJ, Aziz A, Canadian Critical Care Trials Group. A survey of Canadian intensivists' resuscitation practices in early septic shock. Crit Care 2007; 11: R74.

4. Feng $X$, Liu J, Yu M, Zhu $S, X u J$. Protective roles of hydroxyethyl starch 130/0.4 in intestinal inflammatory response and survival in rats challenged with polymicrobial sepsis. Clin Chim Acta 2007; 376: 60-7.

5. Feng $X, H u Y$, Ding $J$, et al. Early treatment with hydroxyethyl starch $130 / 0.4$ causes greater inhibition of pulmonary capillary leakage and inflammatory response than treatment instituted later in sepsis induced by cecal ligation and puncture in rats. Ann Clin Lab Sci 2007; 37: 49-56.

6. Dieterich HJ, Weissmuller T, Rosenberger P, Eltzschig HK. Effect of hydroxyethyl starch on vascular leak syndrome and neutrophil accumulation during hypoxia. Crit Care Med 2006; 34: 1775-82.

7. Marx G, Pedder S, Smith L, et al. Resuscitation from septic shock with capillary leakage: hydroxyethyl starch $(130 \mathrm{kd})$, but not Ringer's solution maintains plasma volume and systemic oxygenation. Shock 2004; 21: 336-41.

8. Dubin A, Pozo MO, Casabella CA, et al. Comparison of $6 \%$ hydroxyethyl starch 130/0.4 and saline solution for resuscitation of the microcirculation during the early goal-directed therapy of septic patients. J Crit Care 2010; 25: 659.e1-8.

9. Guidet B, Martinet $O$, Boulain T, et al. Assessment of hemodynamic efficacy and safety of $6 \%$ hydroxyethylstarch $130 / 0.4$ vs. $0.9 \% \mathrm{NaCl}$ fluid replacement in patients with severe sepsis: The CRYSTMAS study. Crit Care 2012; 16: R94.

10. Magder S, Potter BJ, Varennes BD, Doucette S, Fergusson D, Canadian Critical Care Trials Group. Fluids after cardiac surgery: a pilot study of the use of colloids versus crystalloids. Crit Care Med 2010; 38: 2117-24.

11. Hartog CS, Skupin H, Natanson C, Sun J, Reinhart K. Systematic analysis of hydroxyethyl starch (HES) reviews: proliferation of low-quality reviews overwhelms the results of well-performed meta-analyses. Intensive Care Med 2012; 38: 1258-71.

12. Gattas DJ, Dan A, Myburgh J, et al. Fluid resuscitation with $6 \%$ hydroxyethyl starch (130/0.4) in acutely ill patients: an updated systematic review and meta-analysis. Anesth Analg 2012; 114: 159-69.

13. Schortgen F, Lacherade JC, Bruneel F, et al. Effects of hydroxyethylstarch and gelatin on renal function in severe sepsis: a multicentre randomised study. Lancet 2001; 357: 911-6.

14. Cittanova ML, Leblanc I, Legendre C, Mouquet C, Riou B, Coriat $P$. Effect of hydroxyethylstarch in brain-dead kidney donors on renal function in kidney-transplant recipients. Lancet 1996; 348: 1620-2.

15. Brunkhorst $F M$, Engel $C$, Bloos $F$, et al. Intensive insulin therapy and pentastarch resuscitation in severe sepsis. N Engl J Med 2008; 358: 125-39.

16. Moskowitz DM, Shander A, Javidroozi M, et al. Postoperative blood loss and transfusion associated with use of Hextend in cardiac surgery patients at a blood conservation center. Transfusion 2008; 48: 768-75.

17. Wilkes MM, Navickis RJ, Sibbald WJ. Albumin versus hydroxyethyl starch in cardiopulmonary bypass surgery: a meta- 
analysis of postoperative bleeding. Ann Thorac Surg 2001; 72: 527-33; discussion 534.

18. Rioux JP, Lessard M, De Bortoli B, et al. Pentastarch $10 \%$ $(250 \mathrm{kDa} / 0.45)$ is an independent risk factor of acute kidney injury following cardiac surgery. Crit Care Med 2009; 37: 1293-8.

19. Huter L, Simon TP, Weinmann L, et al. Hydroxyethylstarch impairs renal function and induces interstitial proliferation, macrophage infiltration and tubular damage in an isolated renal perfusion model. Crit Care 2009; 13: R23.

20. Sakr Y, Payen D, Reinhart K, et al. Effects of hydroxyethyl starch administration on renal function in critically ill patients. $\mathrm{Br} \mathrm{J}$ Anaesth 2007; 98: 216-24.

21. Eisenbach $C$, Schonfeld AH, Vogt $N$, et al. Pharmacodynamics and organ storage of hydroxyethyl starch in acute hemodilution in pigs: influence of molecular weight and degree of substitution. Intensive Care Med 2007; 33: 1637-44.

22. Myburgh JA, Finfer S, Bellomo R, et al. Hydroxyethyl starch or saline for fluid resuscitation in intensive care. N Engl J Med 2012; 367: 1901-11.

23. Perner A, Haase N, Guttormsen AB, et al. Hydroxyethyl starch $130 / 0.42$ versus Ringer's acetate in severe sepsis. N Engl J Med 2012; 367: 124-34.

24. Myburgh J, Li Q, Heritier S, Dan A, Glass P, Crystalloid Versus Hydroxyethyl Starch Trial (CHEST). Statistical analysis plan for the Crystalloid Versus Hydroxyethyl Starch Trial (CHEST). Crit Care Resusc 2012; 14: 44-52.

25. Chong PC, Greco EF, Stothart D, et al. Substantial variation of both opinions and practice regarding perioperative fluid resuscitation. Can J Surg 2009; 52: 207-14. 